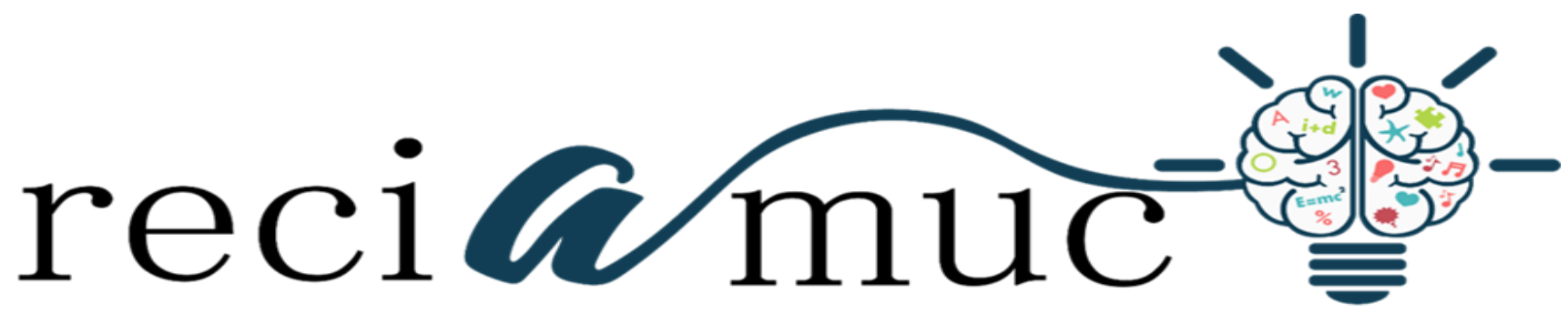

Revista científica de investigación actualización del mundo de las ciencias

Kruzcaya Monserrath Arévalo Sánchez a ; Ronald Roberto Reyes Sánchez ${ }^{\text {; }}$

Marjorie Jacqueline Ramírez Ortiz ${ }^{c}$; Carlos Xavier Villavicencio Bourne ${ }^{d}$

Falla Ovárica en la Osteoporosis

Ovarian Failure in Osteoporosis

Revista Científica de Investigación actualización del mundo de las Ciencias. Vol. 3 núm., 3, julio, ISSN: 2588-0748, 2019, pp. 63-81

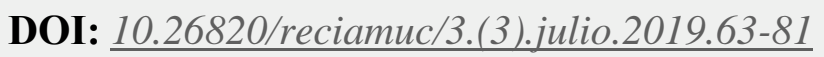

URL: http://reciamuc.com/index.php/RECIAMUC/article/view/267

Código UNESCO: 3205 Medicina Interna

Tipo de Investigación: Artículo de Revisión

(c) RECIAMUC; Editorial Saberes del Conocimiento, 2019

Recibido: 01/06/2019

Aceptado: 05/06/2019

Publicado: 01/07/2019

Correspondencia: kruzcaya.arevalo.md@gmail.com

a. Médico; Investigador Independiente; Guayaquil, Ecuador; kruzcaya.arevalo.md@gmail.com

b. Médico; Investigador Independiente; Guayaquil, Ecuador; triplerrr@ hotmail.es

c. Médico; Investigador Independiente; Guayaquil, Ecuador; elloreal_22@ hotmail.com

d. Medico; Investigador Independiente; Guayaquil, Ecuador; thecharlesone@ hotmail.com 


\section{Falla Ovárica en la Osteoporosis}

Vol. 3, núm. 3., (2019)

Kruzcaya Monserrath Arévalo Sánchez; Ronald Roberto Reyes Sánchez; Marjorie Jacqueline Ramírez Ortiz, Carlos Xavier Villavicencio Bourne

\section{RESUMEN}

La menopausia representa para la mujer un evento fisiológico que aparece entre los cuarenta y sesenta años, con una media a los cincuenta uno. Pero; cuando se manifiesta ante de los cuarenta años, es un trastorno no físiológico, se encuentra asociado a una disfunción ovárica correspondiente a la insuficiencia primaria que los ovarios empiezan caracterizados por una amenorrea de al menos cuatro meses, deficiencias de esteroides sexuales y dos determinaciones de concentración sérica de hormona folículo estimulante. Según Yang (2016) la falla ovárica "afecta a 1 de cada 100 mujeres menores de 40 años (1\%) y 1 de cada 1000 mujeres menores de 40 años $(0,1)$ y 1 de cada 10000 mujeres menores de 20 años $(0,01 \%)$ " (p.15). Cabe agregar que cuando se hace evidente, la presencia de esta falla ovárica, debido a sus características del evento fisiológico, la mujer sufre una serie de consecuencias, siendo una de ellas la osteoporosis, que representa una enfermedad esquelética en la que se produce una disminución de la densidad ósea; es decir, los huesos se vuelven porosos, aumenta el número y tamaño de las cavidades que existen en su interior, haciéndolos más frágiles, resisten poco a los golpes y se rompen con facilidad. Estas evidencias llevan a reflexionar que es importante analizar la falla ovárica en la osteoporosis, elemento esencial para darle la respectiva sustentación teórica al presente artículo, el cual se ubica en el tipo de investigación documental bibliográfico y así permitir luego la elaboración de un cuerpo de conclusiones como apreciaciones generales del tema.

Palabras Claves: Falla Ovárica; Osteoporosis; Menopausia; Densidad Ósea. 
Vol. 3, núm. 3., (2019)

Kruzcaya Monserrath Arévalo Sánchez; Ronald Roberto Reyes Sánchez; Marjorie Jacqueline Ramírez Ortiz, Carlos Xavier Villavicencio Bourne

\begin{abstract}
The menopause for women represents a physiological event that appears between forty and sixty years, with a mean the fifty one. But; when it manifests the forty years before, is a nonphysiological disorder, is associated with ovarian dysfunction corresponding to the primary failure which the ovaries are characterized by at least four months of amenorrhea, deficiencies of sex steroids and two determinations of serum concentration of follicle stimulating hormone. According to Yang (2016) ovarian failure "affects 1 out of 100 women under age 40 (1\%) 1 out of 1000 women under age $40(0.1)$ and 1 out of 10000 women less than 20 years $(0,01 \%) "(p .15)$. It should be added that when it becomes evident, the presence of this ovarian failure, due to its characteristics of the physiological event, the woman suffers a series of consequences, one of them being the osteoporosis, representing a skeletal disease that is It produces a decrease in bone density; i.e., the bones become porous, increasing the number and size of the cavities that exist inside, making them more fragile, they resist little shock and break easily. This evidence lead to think that it is important to analyze the ovarian failure in osteoporosis, essential element to give the respective theoretical support this article, which lies in the type of documentary research library and thus then allow metal works.
\end{abstract}

Key Words: Ovarian Failure; Osteoporosis; Menopause; Bone Density. 


\section{Falla Ovárica en la Osteoporosis}

Vol. 3, núm. 3., (2019)

Kruzcaya Monserrath Arévalo Sánchez; Ronald Roberto Reyes Sánchez; Marjorie Jacqueline Ramírez Ortiz, Carlos Xavier Villavicencio Bourne

\section{Introducción.}

El proceso evolutivo que vive la mujer, presenta una característica determinante que la diferente del hombre, sus órganos sexuales que le permiten desarrollarse en función a su género. Sin embargo, existen respuestas fisiológicas que hacen posible la presencia de enfermedades como la falla ovárica prematura, caracterizada por tener períodos menstruales irregulares ocasionados durante años. Ante dicha problemática, los especialistas buscan que la mujer recupere los niveles de estrógenos, pues, mediante su utilización se logran reducir algunas complicaciones como la osteoporosis, que se produce como consecuencia de los niveles bajos de estrógenos.

En consecuencia, la presencia de la falla ovárica, para su valoración médica amerita identificar el funcionamiento de los componentes del eje hipotálamo-hipófisis ovario; pues, el primero en más o menos cada hora el núcleo arcuato libera la hormona liberadora de gonadotropinas en forma pulsátil al sistema porta hipofisario, lo que estimula la liberación de la hormona folículoestimulante(FSH)yla luteinizante(LH) por la hipófisis. Estas hormonas actúan sobre el ovario en la producción de estrógenos, maduración folicular, ovulación, con el objeto de lograr la reproducción. Esta frecuencia pulsátil del hipotálamo se altera en la premenopausia por cambios en la actividad de las células nerviosas del cerebro.

De lo antes expuesto, se puede indicar que las gonadotropinas actúan sobre el ovario a través de los receptores de membrana. Se conoce que la hormona folículo- estimulante (FSH) estimula la síntesis de receptores para sí misma en la granulosa y para hormona luteinizante (LH) en la teca folicular. El aumento de la LH inhibe la síntesis de receptores para LH. En la falla ovárica precoz (FOP) se ausenta la formación de receptores para gonadotropinas. Este proceso de producción hormonal presente en la mujer conlleva a generar las variaciones hormonales múltiples que se observan durante los días que conforman el ciclo menstrual.

Al respecto, Pacheco (2017), conceptualiza la falla ovárica precoz "como una amenorrea secundaria que se presenta en una mujer antes de los 40 años, conlleva ahipoestrogenismo, infertilidad, menopausia prematura, osteoporosis, enfermedad cardiovascular, trastornos neurovegetativos, entre otros" (p.6). De allí, que la presencia de la falla ovárica, no es más que el 


\section{Falla Ovárica en la Osteoporosis}

Vol. 3, núm. 3., (2019)

Kruzcaya Monserrath Arévalo Sánchez; Ronald Roberto Reyes Sánchez; Marjorie Jacqueline Ramírez Ortiz, Carlos Xavier Villavicencio Bourne

agotamiento folicular, por dotación insuficiente de folículos o destrucción acelerada de los ovocitosy que, debido a este agotamiento folicular debido a dotación insuficiente de folículos, la mujer inicia un proceso caracterizado por la presencia de un ciclo menstrual variable, además de las consecuencias antes citadas por el autor.

De acuerdo, con lo citado por el autor, se precisa que las mujeres que han sido diagnosticadas con falla ovárica, van a tener una serie de consecuencias debido al agotamiento folicular o destrucción acelerada de los ovocitos, esto conduce a la presencia de una amenorrea y por lo tanto, aparecen nuevas enfermedades entre ellas la osteoporosis, vista por Maldonado (2016) como una enfermedad multifactorial generalizada del esqueleto, asintomática, caracterizada por una masa ósea baja y alteración de la micro-arquitectura del hueso, aumento de la fragilidad”.(p.25). Es decir, dicha enfermedad conduce a que la mujer debe

Entonces, la aparición de la osteoporosis como enfermedad multifactorial, representa una consecuencia en las mujeres diagnosticadas de falla ovárica, pues, por ser una enfermedad metabólica ósea común en mujeres que han sido diagnosticadas con falla ovárica, las mismas presentan un aumento del riesgo de facturas; por ello, es importante aplicar medidas para frenar la pérdida de masa ósea, que obliga a las mujeres a realizar ejercicios como medida de control para el avance de la enfermedad. En esta dirección, Bravo (2017) indica que la osteoporosis "se considera una enfermedad metabólica ósea más prevalente estimándose que actualmente afecta al $20 \%$ o $30 \%$ de la población mayor de 50 años". (p.8). Por lo tanto, la existencia de esta enfermedad en las mujeres diagnosticas con falla ovárica, incrementa en ellas, la presencia de un cuidado efectivo en lo que se refiere a su salud, pues, es importante evitar las ciadas, dado que, las mismas generan fracturas y pueden tener consecuencias mayores.

Dentro de este marco de ideas, se visualiza que existe una vinculación en cuanto a la presencia de la osteoporosis en mujeres que presentan falla ovárica, debido al déficit estrogénico que dan lugar, fundamentalmente, a una pérdida de hueso esponjoso, situación que se mantiene en la mujer durante su crecimiento y al presentar la falla ovárica, donde las disminuciones de las hormonas tiroideas afectan la condición ósea de los huesos. Esto se manifiesta como resultado a la esterogenopenia caracterizada por la disminución de los estrógenos y por ende las condiciones 


\section{Falla Ovárica en la Osteoporosis}

Vol. 3, núm. 3., (2019)

Kruzcaya Monserrath Arévalo Sánchez; Ronald Roberto Reyes Sánchez; Marjorie Jacqueline Ramírez Ortiz, Carlos Xavier Villavicencio Bourne

óseas que presenta el hueso se hacen cada vez más frágil. Tal como lo aseveran, Rojas y Bermúdez (2017) "los casos con falla ovárica reportan alrededor del 50\% de las pacientes, que podrán recuperar la función ovárica, pero la existencia de enfermedades como la osteoporosis afecta progresivamente el desarrollo de sus actividades cotidianas”. (p.35)

Asimismo, Escalante (2016) precisa que:

Aproximadamente $1 \%$ de las mujeres menores de 40 años específicamente la menopausia espontánea temprana afecta el 5\% de mujeres en edades entre 40 y 45 años. Destaca que, no se encuentran causas aparentes que condicione la alteración de la función ovárica, porque se clasifica como idiopática $(90 \%)$, en otras condiciones, puede explicarse como una enfermedad autoinmune genética, infecciosa, inflamatoria, iatrogénicas, deficiencias enzimáticas y síndrome metabólicos (p.19)

Por consiguiente, la presencia de la falla ovárica representa un porcentaje significativo en las mujeres a nivel mundial, asimismo, se precisa que, la misma sus causas son poco conocidas y en función de ello la ciencia médica buscan estimar sus consecuencias como una forma de emplear medicamentos especiales que ayuden a las mujeres a tener un nivel de vida. Es decir, mediante la presencia de la insuficiencia ovárica prematura se puede fácilmente estimar que la misma se acompaña de un hipogonadismohipergonadotrofico y amenorrea antes de los cuarenta años de edad.

Estos datos, permiten resaltar la importancia teórica del tema, razón por la cual, se plantean acciones de indagación relativas a encontrar y replantearse nuevas informaciones expuestas mediante valoraciones específicas como un indicador que caracteriza el presente artículo, como parte de un proceso investigativo, donde se analiza la falla ovárica en la osteoporosis, las combinaciones de estos aspectos llevan a la exposición de criterios básicos.

\section{Método.}

La naturaleza del conocimiento científico es elaborar nuevas argumentaciones que ofrezcan oportunidades de cambios en el campo social, político, económico, salud entre otros. Por ello, al 


\section{Falla Ovárica en la Osteoporosis}

Vol. 3, núm. 3., (2019)

Kruzcaya Monserrath Arévalo Sánchez; Ronald Roberto Reyes Sánchez; Marjorie Jacqueline Ramírez Ortiz, Carlos Xavier Villavicencio Bourne

momento de llevar a cabo una actividad enmarcada en la recopilación de informaciones relacionadas con un tema de interés, lleva al investigador planificar acciones pertinentes que le aseguren el empleo del método en correspondencia al contenido seleccionado. Al respecto, Bustillos (2016) define al método como "una herramienta fundamental que ayuda a recoger informaciones sin crear contradicciones entre los planteamientos, todo lo contrario, eleva las características generales de la investigación”. (p.27)

Con respecto a la definición dada por Bustillos, precisa su importancia en el campo de redacción de los contenidos que caracterizan a este artículo, mediante su incorporación se pudo, organizar cada uno de los momentos especiales relevantes para la realización del análisis. En consecuencia, las características temáticas llevan a seleccionar como método el inductivo, visto por el autor antes citado "como el análisis particular de las teorías para luego combinarlo con el todo, para así llegar a una interpretación veraz de los acontecimientos por conocer”. (p.29). Según lo indicado, se puede entender que, a través del método inductivo, se puede primero seleccionar los tópicos para luego generar una nueva apreciación global del fenómeno en estudio.

\section{Tipo de Investigación.}

Los argumentos citados en párrafos anteriores, permiten establecer la respectiva proyección en cuanto a la relación investigativa del artículo con el campo científico, es así como, se hace necesario ubicarlo en el tipo documental, definida por Bello (2017) "trabajos que expresan ideas, tomadas de textos o diferentes materiales impresos, tecnológicos o bibliográficos para luego ampliar su contenido hacia otros escenarios". (p.59). Esta conceptualización, permite valorar que los diferentes argumentos presentes en este artículo, hacen posible redimensionar aquellos aspectos relativos a la falla ovárica en la osteoporosis, eventos esenciales que aseguran de manera explícita las interacciones que pueden ser compaginadas en un momento determinado.

\section{Técnicas Documentales.}

Para el desarrollo del presente artículo, por encontrarse en el campo científico de la investigación documental, razón por la cual, exige incorporar técnicas esenciales para la redacción de los diferentes párrafos que estructuran el contenido temático caracteriza el proceso investigativo. En 


\section{Falla Ovárica en la Osteoporosis}

Vol. 3, núm. 3., (2019)

Kruzcaya Monserrath Arévalo Sánchez; Ronald Roberto Reyes Sánchez; Marjorie Jacqueline Ramírez Ortiz, Carlos Xavier Villavicencio Bourne

esta dirección temática, Bello (ob.cit) destaca que las técnicas documentales "son procedimientos que se utiliza para construir conocimientos, describirlos o dar una explicación a un evento de la realidad de cual no se tiene conocimiento". (p. 62). Según la apreciación, el desarrollo del contenido temático previamente establecido una vez que se selecciona el tema, inicia un procedimiento de actividades inherentes con la revisión de documentos, que hacen posible describir los aspectos individuales para luego construir los aspectos generales del tema.

En consecuencia, para este artículo se consideró pertinente la utilización de diferentes procedimientos enmarcados en la revisión (material bibliográfico y tecnológico) que sirvieron de base para el desarrollo de los diferentes contenidos vinculados con la falla ovárica en la osteoporosis, de allí, la importancia de incorporar la lectura rápida que hizo posible la sistematización de los aspectos que generan nuevos conocimientos para este trabajo. Asimismo, se pudo precisar las ideas principales de cada tema relacionado con el tema, además, de sincronizar los aspectos recabados en las fuentes tecnológicas, hicieron posible generar un análisis preciso de la información obtenida. En otras palabras, fue una revisión exhaustiva de las interpretaciones recabadas para recabar informaciones especiales en cuanto al análisis se refiere, para la presentación del artículo y así generar cambios progresivos en cada uno de los aspectos incorporados.

\section{Técnicas para la Recolección de Información.}

Los aspectos concernientes a la falla ovárica en la osteoporosis, para ser debidamente organizados necesitan obligatoriamente un análisis preciso y coherente en las apreciaciones dadas por los autores al ser valorados para insertarlos en el artículo y así lograr el respectivo desarrollo amplio y profundo del tema indicado anteriormente. Para Bello (ob.cit) durante la redacción de un documento las técnicas para recopilar la información "son básicas en su construcción, las mismas depende de la naturaleza investigativa y son esenciales para dinamizar el trabajo científico". (p. 64). De allí, que la construcción de las ideas complementarias del tema establecido, se hizo necesario incluir el análisis crítico como técnica de interpretación. 


\section{Falla Ovárica en la Osteoporosis}

Vol. 3, núm. 3., (2019)

Kruzcaya Monserrath Arévalo Sánchez; Ronald Roberto Reyes Sánchez; Marjorie Jacqueline Ramírez Ortiz, Carlos Xavier Villavicencio Bourne

Según las apreciaciones anteriores, se puede entender que la conducción interpretativa que determina el desarrollo del artículo, requiere de una actuación crítica del investigador, para lograr su respectiva ubicación en un campo científico y así generar un uso racional de las mismas. Por ello, durante la redacción del contenido seleccionado, se tuvo que recurrir a lecturas básicas como indicador del aporte referencial al tema, luego desglosar sus apreciaciones para finalmente exponer los nuevos contenidos.

\section{Resultados.}

El contenido relacionado con este evento del artículo, amerita el desarrollo de los elementos formadores del texto, en consecuencia, los mismos son organizados en función a las características del fenómeno.

\section{Falla Ovárica.}

La insuficiencia ovárica primaria, también conocida como falla ovárica prematura, ocurre cuando los ovarios de una mujer dejan de funcionar en forma normal antes de cumplir los 40 años. En consecuencia, las mujeres son menos fértiles y comenzar a tener períodos menstruales irregulares a medida que entran a la menopausia. Pero, con insuficiencia ovárica primaria, los períodos irregulares y fertilidad reducida comienzan antes de esta edad indicada, o comenzar hasta en la adolescencia. De este modo, la falla ovárica es diferente a la menopausia prematura, sus períodos se detienen antes de los 40 años y ya no puede quedar embarazada. La causa puede ser natural o puede ser una enfermedad, cirugía, quimioterapia o radiación.

En la insuficiencia ovárica primaria, algunas mujeres todavía tienen períodos ocasionales. Incluso pueden quedar embarazadas. En la mayoría de los casos de insuficiencia ovárica primaria, la causa es desconocida. Cabe agregar que, dicha enfermedad tiene unos signos caracterizados por períodos irregulares o ausencia de ellos, además de otros síntomas como: sofocos, sudores nocturnos, irritabilidad, falta de concentración, dolor durante las relaciones sexuales y sequedad vaginal. Causas, que motivan a buscar un diagnóstico médico. 


\section{Falla Ovárica en la Osteoporosis}

Vol. 3, núm. 3., (2019)

Kruzcaya Monserrath Arévalo Sánchez; Ronald Roberto Reyes Sánchez; Marjorie Jacqueline Ramírez Ortiz, Carlos Xavier Villavicencio Bourne

Por consiguiente, las evaluaciones médicas precisan durante el diagnóstico, al encontrar niveles bajos de ciertas hormonas tiene mayor riesgo de sufrir afecciones específicas, que guardan relación con la producción de las diferentes hormonas encargadas de estimular la producción de óvulos. Al respecto, Calles (2015) expresa que entre las afecciones destaca las siguientes:

- Ansiedad y Depresión: Los cambios hormonales causados por la falla ovárica primaria pueden contribuir a la ansiedad o provocar depresión.

- Síndrome del Ojo Seco y Enfermedad de la Superficie Ocular: Algunas mujeres con insuficiencia ovárica primaria tienen uno de estos problemas de los ojos. Ambos pueden causar incomodidad y visión borrosa. Si no se tratan, estas afecciones pueden causar daño permanente a los ojos.

- Enfermedad del Corazón: Niveles más bajos de estrógeno pueden afectar los músculos que rodean las arterias y aumentar la acumulación de colesterol en ellas. Estos factores aumentan su riesgo de arterosclerosis (endurecimiento de las arterias)

- Infertilidad: Ocasionada por la baja producción de estrógenos o simplemente la disminución de óvulos generados por deficiencias hormonales.

- Hipotiroidismo: Este problema también se conoce como hipofunción tiroidea. La tiroides es una glándula que produce hormonas que controlan el metabolismo y el nivel de energía de su cuerpo. Bajos niveles de hormonas tiroideas pueden afectar su metabolismo y causar que tenga muy poca energía, lentitud mental y otros síntomas.

- Osteoporosis: La hormona estrógeno ayuda a mantener huesos fuertes. Sin suficiente estrógeno, las mujeres con insuficiencia ovárica primaria a menudo desarrollan osteoporosis, una enfermedad ósea que causa huesos débiles y frágiles y que tienen más probabilidades de romperse.

De acuerdo, con las apreciaciones anteriores, se puede decir que actualmente, no existe un tratamiento comprobado para restablecer el funcionamiento normal de los ovarios de una mujer. Pero hay tratamientos para algunos de los síntomas de la insuficiencia ovárica primaria. También hay formas de reducir sus riesgos de salud y tratar las afecciones que puede causar; según Calles (ob.cit) precisa una serie de tratamientos tales como: 
Falla Ovárica en la Osteoporosis

Vol. 3, núm. 3., (2019)

Kruzcaya Monserrath Arévalo Sánchez; Ronald Roberto Reyes Sánchez; Marjorie Jacqueline Ramírez Ortiz, Carlos Xavier Villavicencio Bourne

- Terapia de Reemplazo hormonal: Es el tratamiento más común. Reemplaza el estrógeno y otras hormonas que sus ovarios no producen. Mediante este tratamiento se mejora la salud sexual y disminuye los riesgos de enfermedad cardíaca y osteoporosis. Por lo general, tomará estas medicinas hasta los 50 años de edad; cerca de la edad en que la menopausia comienza.

- Suplementos de Calcio y Vitamina D: Debido a que las mujeres con insuficiencia ovárica primaria tienen un mayor riesgo de osteoporosis, deben tomar calcio y vitaminas todos los días.

- Fertilización in vitro: Si tiene insuficiencia ovárica primaria y desea quedar embarazada, puede considerar la fertilización in vitro.

- Actividad Física regular y un Peso Corporal Saludable: Por ello, es importante la realización de ejercicios regularmente y controlar el peso, para bajar su riego de osteoporosis y enfermedad cardíaca

- Tratamientos para Enfermedades Relacionadas: Si tiene una afección que está relacionada con insuficiencia ovárica primaria, es importante tratarlas también. Los tratamientos pueden incluir medicamentos y hormonas.

Del mismo modo, se puede indicar que la función ovárica anormal va a producir alteraciones de la menstruación, entre ellas: amenorrea y hemorragia uterina disfuncional. La amenorrea puede ser primaria si nunca hubo menstruación, secundaria si es que existió al menos una menstruación en la vida de la mujer y fisiológica, como la que ocurre en el embarazo y lactancia. Mientras tanto, la hemorragia uterina disfuncional puede ser ovulatoria en $10 \%$ de los casos y anovulatoria.La disfunción ovulatoria puede ocurrir en estados hipoestrogénicos e hiperestrogénicos, como se observa en la tabla $\mathrm{N}^{\circ} 1$. Así, se puede entender que la falla ovárica precoz se sitúa en la disfunción ovulatoria en un estado hipoestrogénicohipergonadotrópico, que puede ser por causa genética, iatrogénica, inmunológica, entre otras. 


\section{Falla Ovárica en la Osteoporosis}

Vol. 3, núm. 3., (2019)

Kruzcaya Monserrath Arévalo Sánchez; Ronald Roberto Reyes Sánchez; Marjorie Jacqueline Ramírez Ortiz, Carlos Xavier Villavicencio Bourne

Tabla $\mathbf{N}^{\circ} 1$ Disfunción Ovulatoria de acuerdo a la cantidad de Estrógenos Circulantes

Tabla 1. Disfunción ovulatoria de acuerdo a la cantidad de estrógenos circulantes.

\begin{tabular}{|c|c|}
\hline \multicolumn{2}{|l|}{ Estados hipoestrogénicos: } \\
\hline $\begin{array}{l}\text { - Hipergonadotrópicos: } \\
\text { (falla ovárica precoz) }\end{array}$ & $\begin{array}{l}\text { - edad } \\
\text { - hereditario-genético } \\
\text { - iatrogénico: cirugia, radioterapia, quimioterapia } \\
\text { - inmunológico }\end{array}$ \\
\hline - Hipogonadotrópicos: & $\begin{array}{l}\text { - alteraciones del hipotálamo } \\
\text { - alteraciones de la hipófisis }\end{array}$ \\
\hline \multicolumn{2}{|l|}{ Estados hiperestrogénicos: } \\
\hline - Hipergonodotrópico: & - sindrome de ovario poliquistico \\
\hline - Hipogonadotrópico: & - tumor productor de esteroides \\
\hline
\end{tabular}

Fuente: Calles (ob.cit).

Al interpretar los aspectos citados anteriormente, llevan a destacar que la falla ovárica se encuentra determinada por la presencia de los estados hipoestrogénicos y hiperestrogénicos, que van a caracterizar la insuficiencia ovárica prematura ocurre en una mujer antes de los 40 años de edad. En ella puede existir agotamiento folicular o disfunción folicular, con alteración del mecanismo de maduración folicular. A su vez, el agotamiento folicular puede deberse a una dotación insuficiente de folículos, como ocurre en la disgenesiagonadal pura, o destrucción acelerada de ovocitos, como ocurre en las alteraciones cromosómicas numéricas, alteraciones cromosómicas estructurales, galactosemia, radioterapia, quimioterapia, cirugía, alteraciones inmunológicas y de manera idiopática.

Por otro lado, la alteración del mecanismo de maduración folicular ocurre en ciertas alteraciones inmunológicas, en el déficit de ciertas enzimas, en las anormalidades de las gonadotropinas y el síndrome de ovario resistente, entre otra; consideraciones que se presentan a continuación. 
Vol. 3, núm. 3., (2019)

Kruzcaya Monserrath Arévalo Sánchez; Ronald Roberto Reyes Sánchez; Marjorie Jacqueline Ramírez Ortiz, Carlos Xavier Villavicencio Bourne

Figura $\mathbf{N}^{\circ} 1$ Mecanismos Involucrados en la Falla Ovárica Prematura

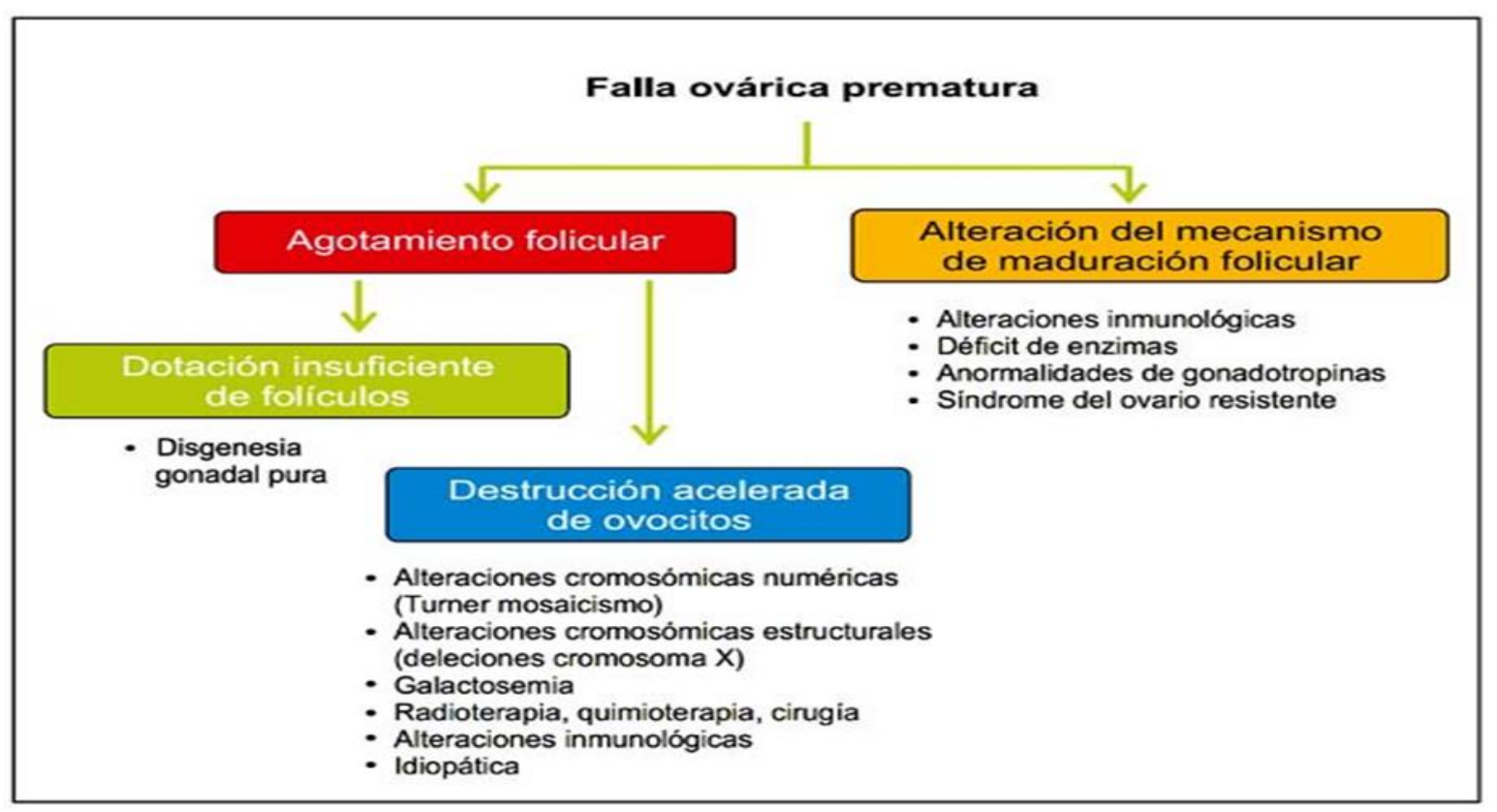

Fuente: Calles (ob.cit)

En líneas generales, la sintomatología que caracteriza a la falla ovárica, es similar a la de la premenopausia, aunque pudiera aparecer más bruscamente y sin síntomas apreciables. Las menstruaciones se vuelven irregulares y van hacia la amenorrea. Puede haber síntomas neurovegetativos, como sudores nocturnos, bochornos y otros, insomnio, irritabilidad, sequedad vaginal, disminución del deseo sexual, aumento del peso corporal, infertilidad. El diagnóstico se realiza en una mujer menor de 40 años con amenorrea más de 3 meses, sintomatología neurovegetativa, signos de hipoestrogenismo. Se incorporará al estudio los valores hormonales en sangre, para determinar la reserva ovárica, encontrándose la FSH mayor de $25 \mathrm{mUL} / \mathrm{mL}$, con disminución de la LH y del estradiol. En la ecografía pélvica, los ovarios muestran volumen disminuido y no se encuentra folículos o son muy escasos. Las condiciones médicas asociadas con esta enfermedad se encuentran otras asociadas como: la osteoporosis, enfermedad cardiovascular, hipotiroidismo, enfermedad de Addison, diabetes mellitus y esterilidad. 


\section{Falla Ovárica en la Osteoporosis}

Vol. 3, núm. 3., (2019)

Kruzcaya Monserrath Arévalo Sánchez; Ronald Roberto Reyes Sánchez; Marjorie Jacqueline Ramírez Ortiz, Carlos Xavier Villavicencio Bourne

\section{Osteoporosis.}

El contenido que caracteriza al segundo término del artículo, lleva a vincularlo especialmente con la falla ovárica, pues, se presenta como una de las consecuencias reportadas durante la evaluación diagnóstica médica, por lo tanto, las apreciaciones se realizan en forma general. La misma, es una enfermedad crónica de afectación ósea que conlleva una menor resistencia esquelética y mayor riesgo de fractura. Cada año, la osteoporosis causa a nivel mundial más de1,3 millones de fracturas vertebrales, muñeca y cadera siendo esta última la más grave que requiere de complejas y costosas intervenciones quirúrgicas. La osteoporosis se considera la enfermedad metabólica ósea más prevalente estimándose que actualmente afecta al 20-30\% de la población mayor de 50 años.

Asimismo, se puede decir que es una enfermedad crónica de afectación ósea. Caracterizada por una disminución de la masa ósea (aspecto cuantitativo) y una alteración de la micro arquitectura (aspecto cualitativo) que compromete la resistencia ósea traduciéndose en una mayor fragilidad ósea y por ende mayor riesgo de sufrir fracturas, forma final de manifestación. La osteoporosis se debe aún desequilibrio en la homeostasia del hueso, puesto que hay un aumento en la resorción y disminución en la formación de hueso.

Cabe destacar que, la presencia de la osteoporosis es más común en edades avanzadas puesto que disminuye la formación ósea asociada a una menor actividad de los osteoblastos. Además, en dicha edad es característico el sedentarismo, una menor exposición solar y por ende menor fuente de vitamina D. Se considera que una mujer de 50 años comienza a experimentar una pérdida contundente de masa ósea, siendo en el hombre a edad de 65-70 años. Por ello, el hecho de ser mujer especialmente en etapa posmenopáusica incrementa el riesgo asociado a una disminución en los niveles de estrógenos con papel protector óseo actuando a través de receptores de estrógenos (ER). Losestrógenos estimulan a los osteoblastos, secretan citosinasantiresortivas e inhiben la apoptosis de los osteoblastos.

Según Bravo (ob.cit) precisa que existen tres tipos principales de osteoporosis

- Osteoporosis Posmenopáusica: Asociada a la menopausia, estado que comienza alrededor de los 51 años ya que en dicho periodo la mujer comienza a perder 
Falla Ovárica en la Osteoporosis

Vol. 3, núm. 3., (2019)

Kruzcaya Monserrath Arévalo Sánchez; Ronald Roberto Reyes Sánchez; Marjorie Jacqueline

Ramírez Ortiz, Carlos Xavier Villavicencio Bourne

progresivamente la función ovárica y por tanto disminuye la producción de hormonas femeninas (estrógenos y progesterona). La falta de estrógenos a esa edad, con papel protector al regular el aporte de calcio en los huesos se asocia con un aumento de la actividad reasortiva. Además, se precisa la actuación de la falla ovárica que como tiene incidencia en la producción de los estrógenos en la mujer, por supuesto, esa disminución conduce a tener como consecuencia la presencia de esta enfermedad.

- Osteoporosis Involutiva o Senil Tipo II: Asociada al envejecimiento con una relación mujer / hombre etapa en la que es característico deficiencia en la actividad de los osteoblastos, un mayor sedentarismo, peor absorción intestinal de calcio, menor exposición solar. Provoca principalmente fracturas de cadera (fémur proximal), dichas fracturas son típicas en la tercera edad.

- Osteoporosis Juvenil Idiopática. Tipo III: Es una enfermedad poco frecuente de causa desconocida que aparece en niños y adultos jóvenes.

- Osteoporosis Secundaria: Son minoritarias y existen múltiples causas, normalmente asociada a otra enfermedad o a un tratamiento farmacológico.

En la mujer menopáusica o en aquellas que presentan falla ovárica se produce un alto remodelado óseo, pérdida de hueso y riesgo de fractura debido al aumento de la actividad osteoclástica, lo que da lugar a una pérdida acelerada de masa ósea que afecta fundamentalmente al hueso trabecular. Ante estas enfermedades, la pérdida de función ovárica y producción de estrógenos, se le atribuye directamente casi el 50\% de la pérdida de masa ósea en la mujer y explica la mayor prevalencia de la osteoporosis en comparación al varón. En efecto, se sabe que los estrógenos disminuyen la actividad osteoclástica inhibiendo la producción de MCSF, RANKL, IL-1, IL-6 y TNF-a, al tiempo que estimulan la producción de OPG y TGF-b, por lo que su déficit tras la menopausia invierte todas estas acciones y condiciona la pérdida acelerada de masa ósea. Además, la resorción ósea exagerada tiende a resultar en hipercalcemia que conlleva a una disminución de la secreción de PTH y de la producción de vitamina $\mathrm{D}$, por lo que disminuye la absorción intestinal de calcio y aumenta la eliminación urinaria del mismo, cerrándose el círculo de las alteraciones fisiopatológicas. 


\section{Falla Ovárica en la Osteoporosis}

Vol. 3, núm. 3., (2019)

Kruzcaya Monserrath Arévalo Sánchez; Ronald Roberto Reyes Sánchez; Marjorie Jacqueline Ramírez Ortiz, Carlos Xavier Villavicencio Bourne

Desde esta visión, se entiende que la falta de estrógenos tiene un efecto agonista en el receptor estrogénico en hueso y antagonista en cerebro y mama. El efecto en el útero puede ser neutral (Raloxifeno) o antagonista en presencia delestrógeno (Bazedoxifeno). Disminuyen la resorción ósea, reducen los marcadores bioquímicos del recambio óseo al rango premenopáusico y aumentan la DMO. Con su uso se pretende, según Baz (2015) la presencia de los estrógenos tiene "efectos beneficiosos sobre el hueso, evita los efectos negativos sobre útero y mama”. (p.13). Es decir, este planteamiento permite entender que cuando las mujeres diagnosticadas con una falla ovárica, disminuye sus estrógenos debido a la disminución de las hormonas y en consecuencia, corren riesgo a sufrir de osteoporosis, afectando con ello la micro arquitectura del hueso. A continuación, se muestra en la figura $\mathrm{N}^{\circ} 2$ el comportamiento de los estrógenos en los huesos.

Figura $\mathbf{N}^{\circ} 2$ Acción de los Estrógenos en los Huesos

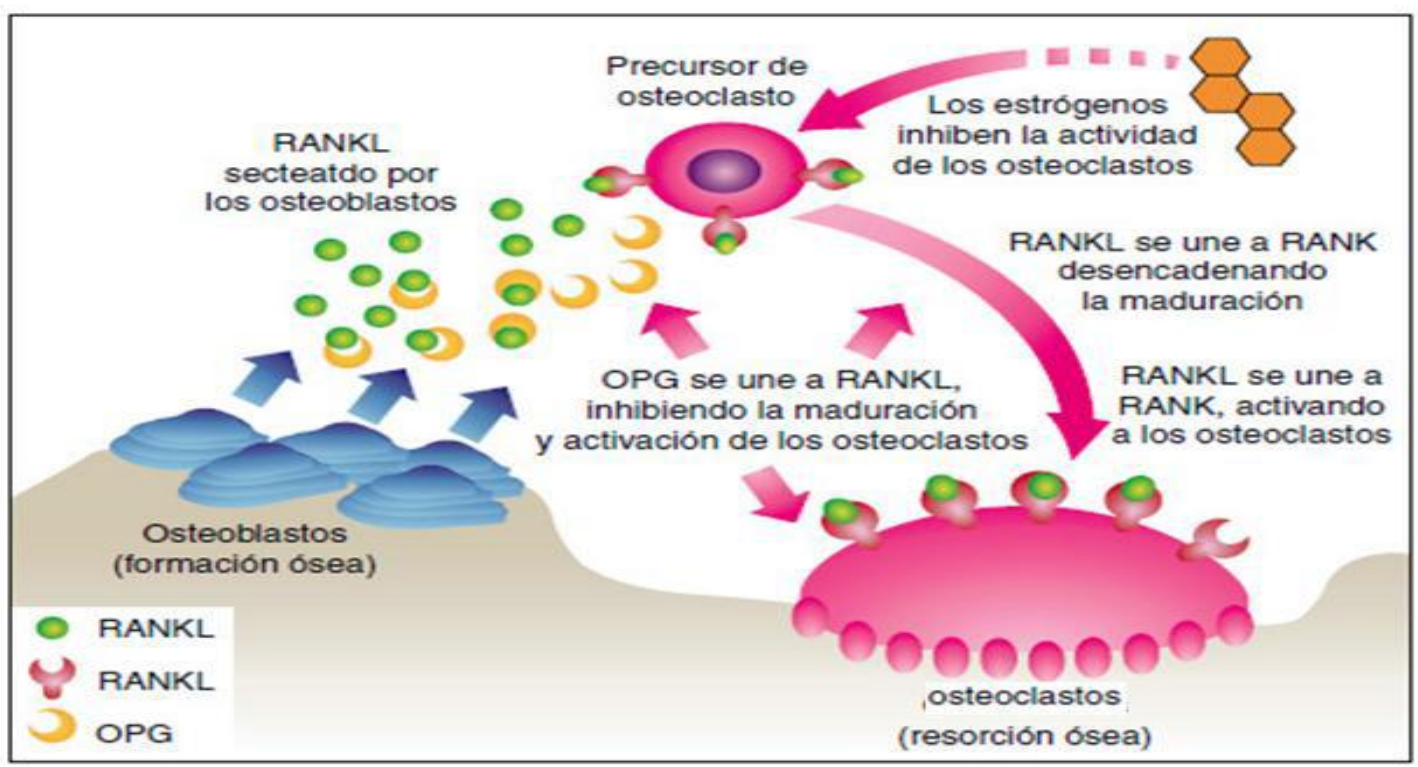

Fuente: Baz (ob.cit)

Por lo tanto, se puede entender que cuando existe una deficiencia de estrógenos en las mujeres, se hace presente la osteoporosis y en consecuencia los huesos se deterioran de forma progresiva, situación que acarrea debilidades y en función de ello, la mujer debe tener una atención médica 


\section{Falla Ovárica en la Osteoporosis}

Vol. 3, núm. 3., (2019)

Kruzcaya Monserrath Arévalo Sánchez; Ronald Roberto Reyes Sánchez; Marjorie Jacqueline Ramírez Ortiz, Carlos Xavier Villavicencio Bourne

integral, encargada de proporcionar los fármacos correspondientes, más aún en aquellas, que presentan falla ovárica, las cuales, al ser joven, el inicio de la osteoporosis a corta edad.

En consecuencia, las causas supuestas de insuficiencia ovárica primaria son varias, lo que ha llevado a crear distintos sistemas de clasificación. En la mayoría de los casos, en el cual la paciente acude a consulta por sus manifiestos síntomas vasomotores sin sospechar que estos síntomas estaban asociados al hipoestrogenismo, lo cual a su vez conllevaría a la osteoporosis de columna y osteopenia de cadera que presenta y que se lo confirmo con los estudios hormonales y densitometría ósea correspondiente. El diagnóstico oportuno en estos casos ayudará a mejorar la calidad de vida, disminuye los riesgos cardiovasculares, de fractura, así como también disminuir la morbimortalidad.

\section{Conclusiones.}

En cuanto a este apartado, es importante considerar los diferentes aportes teóricos e interpretaciones insertadas en el texto correspondiente al tema seleccionado previamente. Entre ella se citan las siguientes:

La presencia de la falla ovárica en las mujeres, tiene una vinculación directa con la producción de estrógenos que el eje del hipotálamo-hipófisis-ovario ocasiona en la mujer una serie de síntomas caracterizada por: períodos menstruales irregulares o salteados (amenorrea), que puede presentarse durante años o desarrollarse después de un embarazo o de dejar de tomar píldoras anticonceptivas. Dificultad para concebir. Sudoraciones nocturnas. Sequedad vaginal. Disminución del deseo sexual, entre otras.

Cabe agregar, que la función ovárica anormal va a producir alteraciones de la menstruación, entre ellas la amenorrea y hemorragia uterina disfuncional. La amenorrea puede ser primaria si nunca hubo menstruación, secundaria si es que existió al menos una menstruación en la vida de la mujery fisiológica, como la que ocurre en el embarazo y lactancia. Entre sus consecuencias van a estar manifestaciones clínicas de depleción de estrógenos y pérdida de la fertilidad. Los niveles bajos de estrógenos se asocian con palpitaciones, intolerancia al calor, bochornos, sudoración nocturna, 


\section{Falla Ovárica en la Osteoporosis}

Vol. 3, núm. 3., (2019)

Kruzcaya Monserrath Arévalo Sánchez; Ronald Roberto Reyes Sánchez; Marjorie Jacqueline Ramírez Ortiz, Carlos Xavier Villavicencio Bourne

irritabilidad, ansiedad, depresión, fatiga, trastornos del sueño, disminución de la libido, cabello grueso, sequedad vaginal.

En una persona joven, la hipoestrogenemia aumenta su riesgo de osteoporosis y trastornos cardiovasculares. Además, el déficit de hormonas sexuales representa un factor de riesgo importante de trastornos neurológicos, metabólicos o cardiovasculares frecuentes y severos, tales como enfermedad de Alzheimer, hipercolesterolemia o enfermedades isquémicas. Por ello, durante el manejo de la falla ovárica, es relevante el uso de terapia estrogénica, aunque no se ha hecho investigación en el tipo de hormona a utilizar, dosis y consecuencias a largo plazo. Tener presente la prevención y manejo de la osteoporosis y las enfermedades cardiovasculares.

Al hacer referencia a la osteoporosis, como enfermedad que afecta la estructura de los huesos y en particular en las mujeres que son diagnosticadas con falla ovárica, se hace presente como resultado a la baja en la producción de estrógenos, hormona esencial para mantener su calidad y condición interna. Pues, el tejido óseo es un órgano vivo constituido por material orgánico y por elementos celulares, en relación al primero se puede indicar el calcio y fosfato constituyen la hidroxiapatita; además de estar presente en ellos elementos inorgánicos con el potasio y bicarbonato.

De lo antes expuesto, se puede indicar que la falla ovárica que manifiestan algunas mujeres antes de los cuarenta años, puede en su edad adulta o mayor sufrir de osteoporosis como resultado a la deficiencia de los estrógenos, es allí, donde se necesita la atención médica dirigida a lograr una evaluación específica mediante estudios de laboratorios y ecografía para estimar los valores de los niveles hormonales y aplicar el respectivo tratamiento, esta prevención logra ofrecerle a las mujeres una mejor calidad de vida.

\section{Bibliografía.}

Baz, T. (2015). Osteroporosis Prevención y Tratamiento en la Mujer. Barcelona : Morata .

Bello, M. (2017). Procedimientos Metodológicos . España : Gedisa .

Bravo, O. (2017). Estudio Comparativo de la Actividad Ovárica . Combia Médica , 5-9. 
Falla Ovárica en la Osteoporosis

Vol. 3, núm. 3., (2019)

Kruzcaya Monserrath Arévalo Sánchez; Ronald Roberto Reyes Sánchez; Marjorie Jacqueline

Ramírez Ortiz, Carlos Xavier Villavicencio Bourne

Bustillos, J. (2016). Investigación Documental . Caracas : SYPAL.

Calles, R. (2017). Síntomas y Causas de la Falla Ovárica . Publicaciones Médicas , 30-39.

Escalante, P. (2016). Diagnóstico Preventivo de la Falla Ovárica . AMA, 30-35.

Maldonado, C. (2016). Estructura Orgánica del Hueso . México: Alfaril .

Pacheco, J. (2017). Síntomas de la Falla Ovárica . IMBIOMED, 4-6.

Rojas, C. y. (2017). Análsisi de la Falla Ovárica . Mexicana Familiar , 31-37.

Yang, L. (2016). Deficiencia Ovárica . Publicaciones Médicas , 15-18.

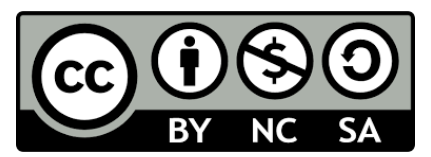

RECONOCIMIENTO-NOCOMERCIAL-COMPARTIRIGUAL

CC BY-NC-SA

ESTA LICENCIA PERMITE A OTROS ENTREMEZCLAR, AJUSTAR Y CONSTRUIR A PARTIR DE SU OBRA CON FINES NO COMERCIALES, SIEMPRE Y CUANDO LE RECONOZCAN LA AUTORÍA Y SUS NUEVAS CREACIONES ESTÉN BAJO UNA LICENCIA CON LOS MISMOS TÉRMINOS. 